\title{
Evaluation of Premature Contacts Using the T-Scan III
}

\author{
Duygu Kürklüarpacay¹, Funda Bayindir², Nuran Dinçkalyanikoğlu² \\ ${ }^{1}$ Faculty of Dentistry, Department of Prosthodontics, Izmir Democracy University, Karabağlarİzmir, Turkey \\ ${ }^{2}$ Faculty of Dentistry, Department of Prosthodontics, University of Atatürk, Erzurum, Turkey \\ Email: dt.duyguk@hotmail.com,duygu.arpacay@idu.edu.tr, fundabayindir@gmail.com,nyanikoglu@gmail.com
}

How to cite this paper: Kürklüarpacay, D., Bayindir, F. and Dinçkalyanikoğlu, N. (2019) Evaluation of Premature Contacts Using the T-Scan III. Open Journal of Stomatology, 9, 136-145.

https://doi.org/10.4236/ojst.2019.95014

Received: March 8, 2019

Accepted: May 27, 2019

Published: May 30, 2019

Copyright (c) 2019 by author(s) and Scientific Research Publishing Inc. This work is licensed under the Creative Commons Attribution International License (CC BY 4.0).

http://creativecommons.org/licenses/by/4.0/

(c) (i) Open Access

\begin{abstract}
Objective: The aim of study is to evaluate premature contacts and occlusal forces for each tooth in complete dentures before occlusal adjustment. Material and methods: The study population was comprised of 30 subjects (aged 50 - 75). The measurements were performed using the T-Scan. The occlusion was analyzed before and after frames which from the T-scan "Force Movies" are Centric Relation bite recordings. Several practices closures were made until a repeatable pattern of contacts was seen on the video monitor and sensor was calibrated. Each subject was asked to bite on the sensor in a position of maximum intercuspation thus obtaining the location of each tooth contact. Premature contacts and percentage distribution of forces per tooth were obtained before and after occlusal adjustment. Results: The worst occlusal balance was recorded in a patient $(\mathrm{F} / 74)$ by occlusal force values at right $5.9 \%$ and left $94.1 \%$ before occlusal adjustment. After occlusal adjustment, all patients' percentage of occlusal balance values was adjusted at average of $48.8 \%$, left $51.1 \%$. The percentage distribution of forces per tooth results showed that premature contacts had been observed at posterior teeth either right or left side of occlusion. Conclusions: Occlusal adjustment is requested for all complete dentures to prevent patients from muscular pain and/or temporomandibular disorders for further terms.
\end{abstract}

\section{Keywords}

Premature Contacts, Occlusal Balance, T-Scan

\section{Introduction}

For the successful prosthetic treatment of edentulous patients, correct occlusal relationships are important. Occlusal checking is needed to be demonstrated by a remount procedure due to the fact that dimensionally accurate complete den- 
tures cannot be provided by denture base materials and fabrication procedures [1].

Complete edentulism not only hampers the mastication, esthetics, but also affects the psychological well-being of the patient. There has been much controversy about various concepts and the theories put forward to achieve occlusion [2].

The concept of bilaterally balanced occlusion, established by Gysi who helped for the progress of scientific knowledge on occlusion, is also a completely balanced occlusion which was created in order to stabilize complete dentures [3].

Pound [4] suggested that the occlusal fossa of the lower jaw is the control point for the balanced occlusion.

Many various factors can cause some problems related to the occlusal relationships of teeth on complete dentures. Unstable trial bases for the interarch relationship record, incorrect use of facebow, transfer of casts on an articulator, inaccurately established vertical and horizontal dimensions, irregular arrangement of posterior teeth, excessive use of pressure during pressing of the acrylic resin into the flask, inadequately closed flask during polymerization, and overheating of the finished dentures during final polishing, can be counted as those factors [1].

The uniform loading of denture foundations and their stability during denture wearing are quite affected by the relationship among the artificial teeth on complete dentures. When dentures are introduced to patients for the first time, clinicians generally prefer a direct determination of occlusion and grinding of denture teeth in the mouth. After the complete dentures, the patient's demand for comfort, function, and esthetics should be satisfied by the occlusion. The influences of the tooth contacts are essential and important for the stability of denture bases, masticatory efficiency, and the transfer of masticatory forces to the supporting areas. Among the methods of correcting occlusal contacts, the one that is difficult and imprecise can be defined as direct grinding of occlusion [1].

Edentulous patients cannot feel prematurities or imbalance of the dentures during process due to the lack of the periodontal ligaments [5]. Difficulties in achieving a reliable occlusal balance include plaster models without tissue resilience and articulators with only functional movements [6]. In the evaluation of occlusal relationships, both qualitative and quantitative methods are preferred to be used [7]. In qualitative analysis, wax, silk strips and articulating paper are used. Since the width, thickness, and dye type of the articulating paper make it able to leave a mark of either a point or a surface, articulating paper is frequently preferred. However, there are major disadvantages of articulating papers which result in huge numbers of pseudo contact markings and can be mentioned as being easily ruined by saliva, being thick, and having relatively inflexible base material [7] [8].

On the other hand, the quantitative method of evaluating occlusal relationships such as Photo-occlusion and T-Scan system (Tekscan Inc., South Boston, MA, USA) provides the differentiation of the sequence and density of the con- 
tacts. Also digital analysis gives additional information for occlusal contact pattern, which includes the quantification of force, sequence of contact, and occlusal-disocclusal timing. Computerized occlusal analysis methods have been developed over time to determine occlusal pressure detection, parafunctional forces, occlusal contacts and contact timing. In diagnosing occlusal problems, identifying occlusal contact force and time aberrances, and guiding the operator through occlusal adjustments during prosthetic and implant dentistry insertions, this system is preferred to use [9] [10] [11]. Several studies have been done with T-scan in occlusal alignment [6] [12] [13]. The T-Scan system was a way to evaluate the distribution of time and force in occlusal balance [13].

In 1987, the T-Scan Occlusal Analysis system manufactured by Tekscan, Inc. (South Boston, MA, USA) was developed by Professor William L. Maness in partnership with M.I.T. [9].

The T-Scan III system consists of a hand-held device with a USB port to be connected to a laptop or a Windows-based PC; the hand-held device contains a $\mathrm{U}$-shaped pressure-measuring sensor that fits into the patient's mouth between the occluding teeth. The pressure-measuring sensor is a grid-based, Mylar-encased recording sensor that is $60 \mu \mathrm{m}(0.06 \mathrm{~mm})$ thick and consists of 1500 compressible sensitive receptor points made of conductive ink. When the patient bites on the sensor, the electrical resistance of the conductive sensor is lessened, since the force applied compresses the particles together; this is recorded as quantitative force data [10] [11] [14].

It records the sequence of occlusal contacts from the first point of contact to maximum intercuspation (MIP), which can be seen as a movie in real-time on the computer screen to analyze occlusal contact information [15].

The reported advantages to indicate accurately occlusal contacts makes the computerized occlusal analysis system a better occlusal indicator when compared with other non-digital conventional indicator materials available [16].

The optical arrangement of the TMJ, disk and muscles is known as the centric relationship. Centric occlusion, on the other hand, can be defined as the mandibular position in which the condyles are in centric relation whereas the denture teeth are in maximal intercuspal position [17]. On denture teeth, the errors in centric occlusion are part of occlusal errors. Removal of occlusal disorders is an important treatment for mandibular dysfunction and Occlusal corrections are effective in cranio-mandibular disease healing [18] [19].

This study has aimed to detect the premature contacts of complete dentures. when the premature contacts were identified, occlusion was arrenged through grinding denture teeth and meanwhile occlusal force was put into a balance. The hypothesis of this study; and that the occlusion arrangement is good for the distribution of occlusal forces and stability of the prosthesis.

\section{Clinical Implications}

Most of premature contacts has been observed at posterior teeth either right or 
left side of occlusion in complete dentures. Computerized occlusal analysis is the best way to do the right occlusal adjustment for complete dentures to understand the first premature contacts.

\section{Material and Methods}

In this study, thirty patients who are completely edentulous preferring Ataturk University, Faculty of Dentistry, Department of Prosthodontics to have constructed new complete dentures from November 2011 to February 2012.Patients were agreed to be worked with and The Ethics Committee of the University of Atatürk has given an approval to the protocol. The procedures followed were in accordance with the ethical standards of the responsible committee on human experimentation (institutional and national) and with the Helsinki Declaration of 1975, as revised in 2000 and 2008 (Ataturk University, Ethic Committee of Faculty of Dentistry, 12.09.2011/013).

The eligibility criteria were as follows: 1) Edentulous maxilla and mandible for at least 3 months at the time for prosthetic treatment. 2) adequate understanding of written Turkish to respond to the questionnaires, and 3) age greater than 50 years. The exclusion criteria were: 1) uncontrolled systematic disease containdicating prosthetic treatment. 2) infectious disease, 3) temporomandibular joint dysfunction 4) orofacial pain. The patients were examined clinically and radiologically before treatment. Basic demographic data of the thirty patients were shown in Figure 1. After treatment procedure finished, all complete dentures were reviewed in respect to stability, function, esthetic. Occlusion of dentures were performed via T-Scan ${ }^{\circledR}$ III (T-Scan III for Windows, Tekscan Inc.). The occlusion was analyzed in before and after frames which from the T-Scan "Force Movies" are Centric Relation (CR) bite recordings.

Several practices closures were made until a repeatable pattern of contacts was seen on the video monitor and sensor was calibrated. The eventuating of "force

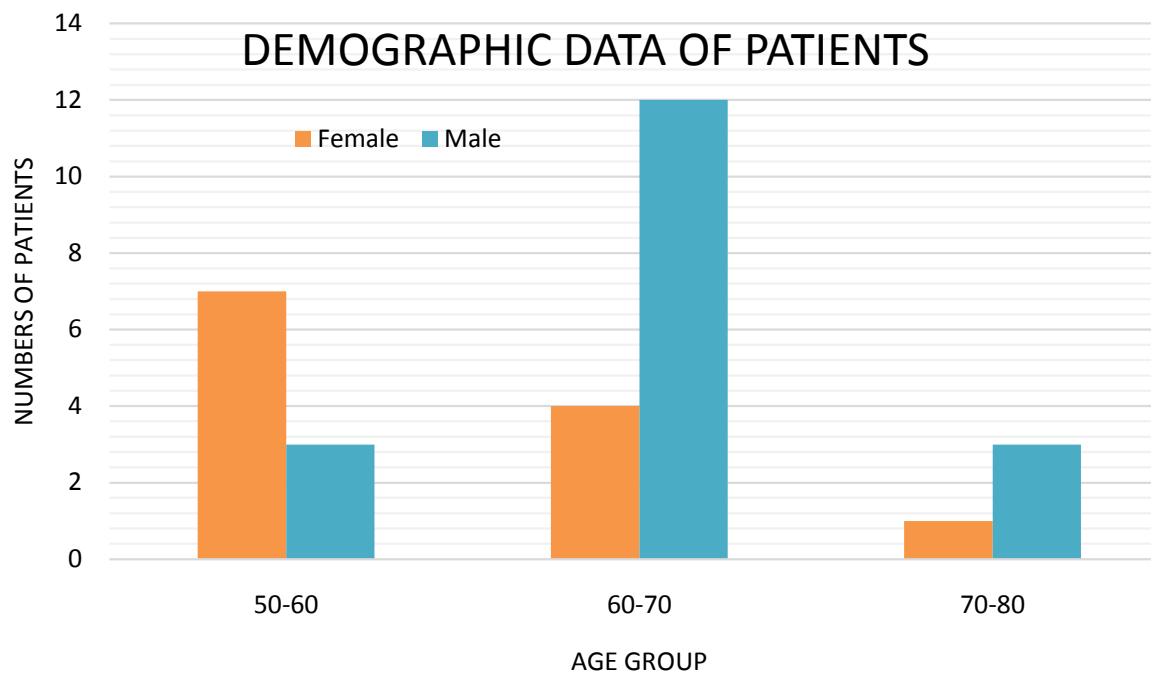

Figure 1. Basic demographic data of patients. 
Movie" indicates contacts of tooth, where bar height and color were used as indicators to identify the forces. When before and after the frames recorded loading and the balance between the occlusal forces and T-Scan's red "center of force (COF)" icon was explained exactly (Figure 2).

T-Scan III analyzes the order of the occlusal contacts while simultaneously measuring the force percentage changes of those same contacts, from the moment the teeth first begin making occlusal contact, all the way through to maximum intercuspation (Figure 3). According to the force percentage values, the highest premature contact tooth was obtained.

The heavy contacts in the centric occlusal position had been corrected by grinding the fossae and inclines of the cusp (Figure 4). This procedure was repeated until the occlusal force was balanced as seen in the bite recordings before

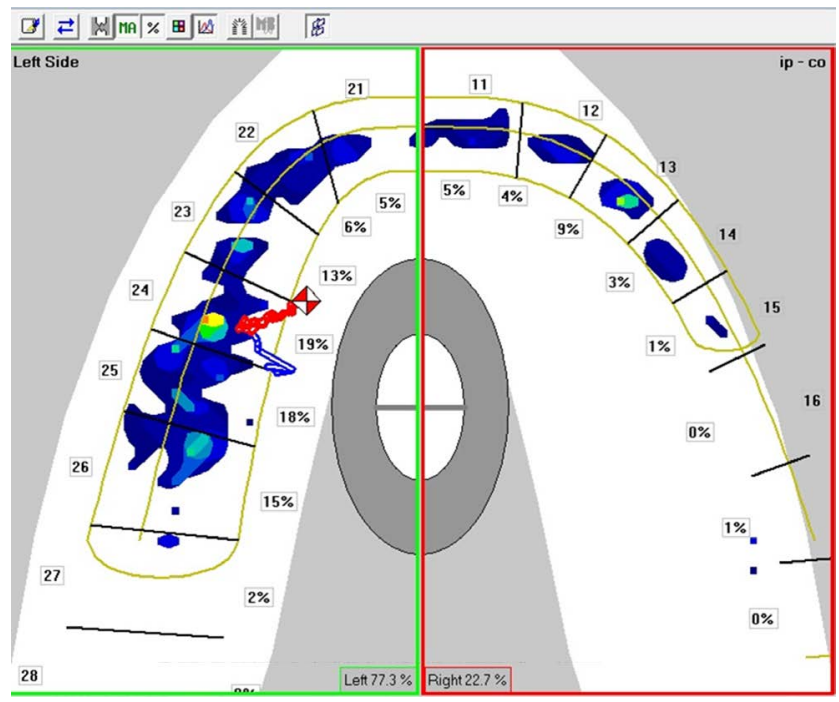

Figure 2. The screen of the T-Scan centric relation bite recording before occlusal adjustment.

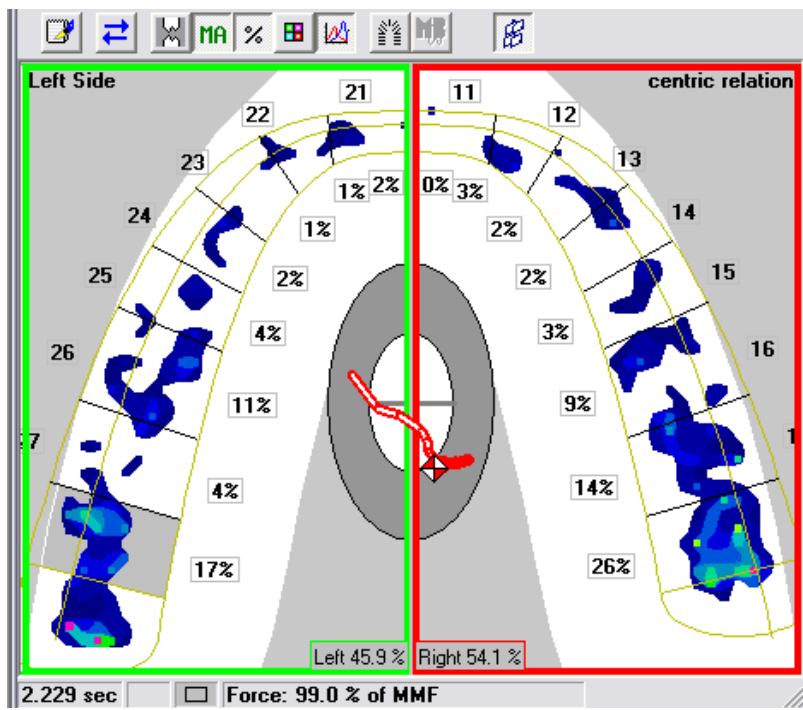

Figure 3. The screen of the T-Scan centric relation bite recording after occlusal adjustment. 


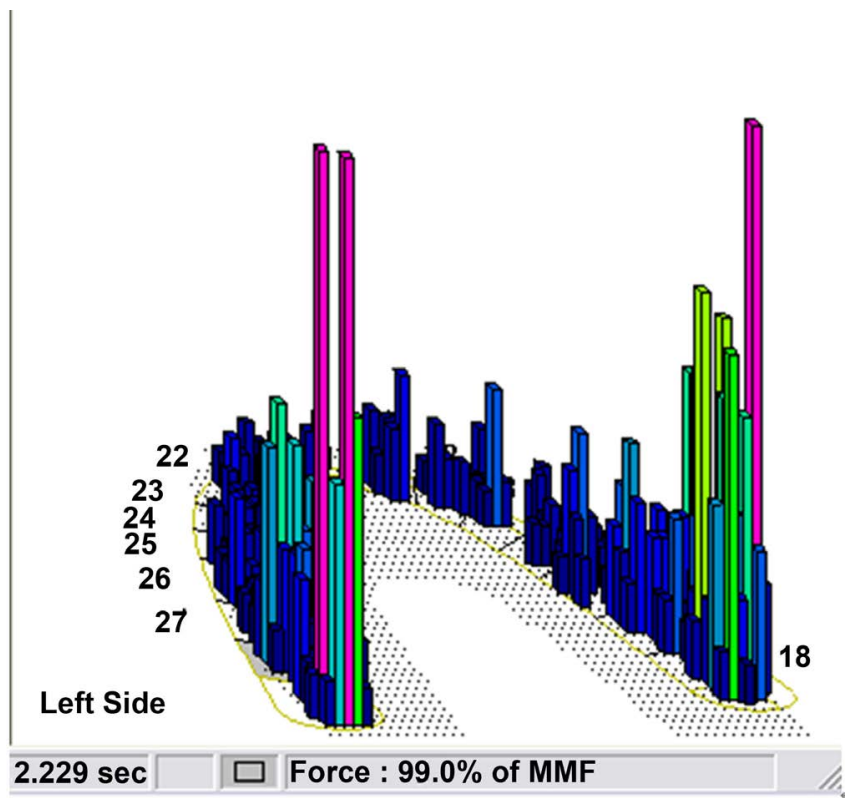

Figure 4. The color and height of each bar indicate the intensity of force per contact.

providing the denture to patients mouth. By grinding the premature contacts, the facets of the upper and lower teeth, which are in harmony with the patient's mandibular movements, can be established. Simultaneously the cuspal inclines may be reduced and some occlusal freedom can be produced. In elderly patients, the mandibular joints are loose and the muscles of mastication's function poorly. Therefore even thought the correct centric occlusal position has been obtained, the position is unstable, so providing some occlusal freedom is essential.

The major limitation of the present study is te rather low number of patients mainly due to the inclusion \& exclusion criteria and the hard cooperation with patients during several measurements. Another limitation may be the lack of blinding to the study group during the measurement of the occlusion as one researcher performed the dentures and occlusal adjusting in all patients.

\section{Results}

The worst occlusal balance was recorded in a patient (F/74) by occlusal force values at right $5.9 \%$ and left $94.1 \%$ before occlusal adjustment. After occlusal adjustment, all patients' percentage of occlusal balance values were adjusted at average right $48.8 \%$, left $51.1 \%$.

The most of premature contacts was detected at posterior teeth either right or left side of occlusion.

\section{Discussion}

In this study, most of premature contacts has been observed at posterior teeth and occlusal adjustment had done by T-scan computer analysis system. The T-Scan III helps us to measurably adjust and create a balanced force distribution between the left and right arch halves. Successful occlusal adjustment provides 
balanced distrubition of occlusal forces and enhances the stability of dentures. Therefore, the null hypothesis was accepted.

After the construction of complete dentures, occlusion must be checked by clinician meticulously because the occlusion of complete dentures is an effective factor for the correct distribution of masticatory loading on the denture foundation, the stability of the denture bases, and the certain extent of patient satisfaction [5].

Tooth contacts may simultaneously occur on all surfaces in all directions and mandibular movements [3]. The lingualized occlusion forms reduced contacts on the palatal cusps in the case of the presences of the fissures of mandibular teeth. Thus, the buccal cusps are not part of contact, and during masticatory function the pressure is lingually transferred to the mandibular complete denture [4]. Monoplane occlusion has occlusal contacts of the lower and upper teeth in maximum intercusputation, disclusion of posterior teeth due to their arrangement in a single plane and contacts of anterior during mandibular movements [20].

According to Stallard, [21] bilateral occlusion is stated as inadequate preference for both natural teeth and complete denture teeth. Therefore the most recent applicable concept for the arrangement of denture teeth in complete dentures is preferred as anterior/canine guidance, which was introduced by Schwab and Stuart and later modified by Gausch [22] and Gutowski [23]. Canine-guided (protected) occlusion figures uniform contacts of premolars and molars in maximal intercuspal position, while the intercanine area is out of contacts. The characteristics of lingualized occlusion are shown in the arrangement and mutual contacts of posterior teeth, which provides for better transfer of masticatory pressure onto the edentulous alveolar ridge and better stabilization of mandibular and maxillary complete dentures as well.

A majority in dental practitioners think that occlusal disturbances are the cause of or closely related with the temporomandibular disorders (TMD), and thus many of the practioners suggest occlusal adjustment as an initial treatment for TMD. In a study, occlusal adjustment results showed a significant reduction in dysfunction and pain symptoms in patients who have headache and show mild TMD symptoms [18]. In a study with acceptable design where 64 TMD patients were treated with occlusal adjustment, performed in one session, and compared with controls of non-treated patients, significant reduction was observed in subjective pain symptoms which was recorded by questionnaire [19].

The final correction of possible occlusal disharmony on dentures should be carried out via the procedure of selective grinding [24].

The time when remounting should be performed is after polymerization of denture bases and before complete dentures are provided to patients. However, the best timing of remounting after optimal incorporation of the denture into the patient's mouth is still not certain.

The timing is determined as 8 to 10 days after the time when the complete 
denture is given to the patient [25] whereas Dapprich and Oidtman [26] suggested for a 2-week time limit after the wearing of the complete denture. In this study occlusal grinding were performed at the last dental session.

For the purpose of presenting computer-guided occlusal force-finishing corrective adjustments that give a serious help for the progress of the installed prosthetic occlusal balance, T-Scan III system can be preferrred in the use with removable prostheses [6].

$\mathrm{Hu}$ et al. [12] stated that occlusal balance was evaluated by T-Scan II system and occlusion of biting of normal subjects with maximal force was stable and bilaterally balanced in intercuspal position. Therefore in this study fabricated complete dentures were evaluated and occlusal adjustment were needed for balanced occlusion.

Mizui et al. [13] suggested that Sixty normal subjects recorded bilateral balance and an anteroposterior center of force in the first molar region. However, patients with craniomandibular disorders showed remarkable differences rather than the control group. As a result, the T-Scan system was found clinically useful in terms of diagnostic screening method for occlusal stability in intercuspal position. In this study T-Scan system was used and provided convenient to measure occlusal forces during adjusting period.

Remounting can be helpful in reducing, preventing changes in occlusion and improving denture comfort as well as the efficiency of occlusal rehabilitation of edentulous patients.

Joanna et al. Stated [27] that The grinding and reconstruction of artificial tooth surfaces and the occlusal vertical dimension affect the force distribution in the centric occlusion and the continuity of the occlusal parameters.

The T-SCAN system provides the only accurate way to determine and evaluate the time sequence and force of occlusal contacts by converting the qualitative data into quantitative and displaying them digitally. Using the system, multiple corrections of imbalanced forces can be performed for every segment of the dentition [28].

\section{Conclusions}

According to the results of this study;

1) The worst occlusal balance was recorded in a patient (F/74) by occlusal force values at right $5.9 \%$ and left $94.1 \%$ before occlusal adjustment.

2) After occlusal adjustment, all patients' percentage of occlusal balance values was adjusted at average $48.8 \%$, left $51.1 \%$.

\section{Acknowledgements}

Supported by Project No: 2005/99 from Scientific Research Project of Ataturk University.

Presented at $101^{\text {st }}$ FDI Annual World Dental Congress in 28-31 August 2013 İstanbul/Turkey. 


\section{Conflicts of Interest}

The authors declare no conflicts of interest regarding the publication of this paper.

\section{References}

[1] Badel, T., Panduric, J., Kraljevic, S. and Dulcic, N. (2007) Checking the Occlusal Relationships of Complete Dentures via a Remount Procedure. The International Journal of Periodontics and Restorative Dentistry, 27, 181-192.

[2] Rangarajan, V., Gajapathi, B., Yogesh, P.B., Mohamed Ibrahim, M., Ganesh Kumar, R. and Karthik, P. (2015) Concepts of Occlusion in Prosthodontics: A Literature Review, Part I. The Journal of Indian Prosthodontic Society, 15, 200-205. https://doi.org/10.4103/0972-4052.165172

[3] Gysi, A. (1929) Practical Application of Research Results in Denture Construction. The Journal of the American Dental Association, 16, 199-223. https://doi.org/10.14219/jada.archive.1929.0056

[4] Pound, E. (2006) Utilizing Speech to Simplify a Personalized Denture Service. Journal of Prosthetic Dentistry, 95, 1-9. https://doi.org/10.1016/j.prosdent.2005.10.004

[5] Palla, S. (1997) Occlusal Considerations in Complete Dentures. In: McNeill, C., Ed., Science and Practice of Occlusion, Quintessence, Chicago, 457-467.

[6] Kerstein, R.B., Thumati, P. and Padmaja, S. (2013) Force Finishing and Centering to Balance a Removable Complete Denture Prosthesis Using the T-Scan III Computerized Occlusal Analysis System. The Journal of Indian Prosthodontic Society, 13, 184-188. https://doi.org/10.1007/s13191-013-0287-1

[7] Schelb, E., Kaiser, D.A. and Brukl, C.E. (1985) Thickness and Marking Characteristics of Occlusal Registration Strips. Journal of Prosthetic Dentistry, 54, 122-126. https://doi.org/10.1016/S0022-3913(85)80086-X

[8] Reiber, T., Fuhr, K., Hartmann, H. and Leicher, D. (1989) Recording Pattern of Occlusal Indicators. I. Influence of Indicator Thickness, Pressure, and Surface Morphology. Deut Zahnaerztl Z, 4, 90-93.

[9] Maness, W.L., Benjamin, M., Podoloff, R., Bobick, A. and Golden, R.F. (1987) Computerized Occlusal Analysis: A New Technology. Quintessence International, 18, 287-292.

[10] Harvey, W.L., Osborne, J.W. and Hatch, R.A. (1992) A Preliminary Test of the Replicability of a Computerized Occlusal Analysis System. Journal of Prosthetic Dentistry, 67, 697-700. https://doi.org/10.1016/0022-3913(92)90174-9

[11] Olivieri, F., Kang, K.H., Hirayama, H. and Maness, W.L. (1998) New Method for Analyzing Complete Denture Occlusion Using the Center of Force Concept: A Clinical Report. Journal of Prosthetic Dentistry, 80, 519-523. https://doi.org/10.1016/S0022-3913(98)70025-3

[12] Hu, Z.G., Cheng, H., Zheng, M., Zheng, Z.Q. and Ma, S.Z. (2006) Quantitative Study on Occlusal Balance of Normal Occlusion in Intercuspal Position. Chinese Journal of Stomatology, 41, 618-620.

[13] Mizui, M., Nabeshima, F., Tosa, J., Tanaka, M. and Kawazoe, T. (1994) Quantitative Analysis of Occlusal Balance in Intercuspal Position Using the T-Scan System. The International Journal of Prosthodontics, 7, 62-71.

[14] Kerstein, R.B. and Grundset, K. (2001) Obtaining Measurable Bilateral Simultane- 
ous Occlusal Contacts with Computer-Analyzed and Guided Occlusal Adjustments. Quintessence International, 32, 7-18.

[15] Kerstein, R.B. (2001) Current Applications of Computerized Occlusal Analysis in Dental Medicine. General Dentistry, 49, 521-530.

[16] Afrashtehfar, K.I. and Qadeer, S. (2016) Computerized Occlusal Analysis as an Alternative Occlusal Indicator. Cranio, 34, 52-57. https://doi.org/10.1179/2151090314Y.0000000024

[17] The Academy of Prosthodontics (1999) The Glossary of Prosthodontic Terms: 7th Edition. Journal of Prosthetic Dentistry, 81, 39-110. https://doi.org/10.1016/S0022-3913(99)70234-9

[18] Forssell, H., Kirveskari, P. and Kangasniemi, P. (1986) Effect of Occlusal Adjustment on Mandibular Dysfunction. A Double-Blind Study. Acta Odontologica Scandinavica, 44, 63-69. https://doi.org/10.3109/00016358609041309

[19] Vallon, D., Ekberg, E.C., Nilner, M. and Kopp, S. (1991) Short-Term Effect of Occlusal Adjustment on Craniomandibular Disorders Including Headaches. Acta Odontologica Scandinavica, 49, 89-96. https://doi.org/10.3109/00016359109005892

[20] Jones, P.M. (1972) The Monoplane Occlusion for Complete Dentures. The Journal of the American Dental Association, 85, 94-100. https://doi.org/10.14219/jada.archive.1972.0293

[21] Stuart, C. and Stallard, H. (1963) Concepts of Occlusion-What Kind of Occlusion Should Recusped Teeth Be Given? Dental Clinics of North America, 7, 591-600.

[22] Gausch, K. (1986) Experiences with Anterior/Cuspid-Controlled Complete Dentures. Deut Zahnaerztl Z, 41, 1146-1149.

[23] Gutowski, A. (1970) Significance of Occlusion in Prosthetic Procedures. Zwr, 79, 8-14.

[24] Zarb, G.A. and McGinney, G.P. (1997) Completing the Rehabilitation of the Patient. In: Zarb, G.A., Bolender, C.L. and Carlsson, G.E., Eds., Boucher's Prosthodontic Treatment for Edentulous Patients, Mosby, St. Louis, 358-389.

[25] Gutowski, A. (1996) Die Remontage von Totalprothesen. Phillip Journal, 13, 79-88.

[26] Dapprich, J. and Oidtmann, E. (2001) Totalprothetik. Klinik und Technik der weiterentwickelten Lauritzen-Methode. Quintessenz, Berlin.

[27] Joanna, K., Teresa, S. and Maria, G. (2017) Evaluation of Functional Parameters in the Occlusion of Complete Denture Wearers before and after Prosthetic Treatment. Journal of Prosthodontic Research, 61, 480-490. https://doi.org/10.1016/j.jpor.2017.03.003

[28] Bozhkova, T.P. (2016) The T-SCAN System in Evaluating Occlusal Contacts. Folia Medica, 58, 122-130. https://doi.org/10.1515/folmed-2016-0015 